CIENCIA Y SOCIEDAD

Volumen XIV, Número 3

Julio - Septiembre, 1989

\title{
EFECTOS DE LECTUCA SATIVA, ANNONA MURICATA Y TAMARINDUS INDICA SOBRE LA CONDUCTA EXPLORATORIA DE RATONES
}

José R. Albaine Pons

\section{Resumen}

Se investigó la conducta de 200 ratones en la prueba de campo abierto, bajo los efectos de inyecciones (ip) de extractos de hojas frescas de Lectuca sativa, Tamarindus indica y Annona muricata a distintas concentraciones. Los resultados indican un efecto inhibidor sobre la locomoción, investigación y acicalamiento, mostrando en este último una diferencia entre $\mathbf{L}$. sativa y el resto, lo que hace posible señalar la función de L. sativa como ansiolítica y la de T. indica y A. muricata, más bien como neuroléptica.

\section{Introducción}

La necesidad del conocimiento de la fitoterapia tradicional en los países del Tercer Mundo está siendo avalada tanto por organismos internacionales, como por especialistas de países desarrollados. ${ }^{2}$

Entre otras cosas se plantea que la "medicina occidental" o científica puede encontrar en plantas medicinales de uso común importantes principios activos que pueden reelaborarse o sintetizarse y pasar al arsenal de la medicina moderna. ${ }^{3}$

Facultad de Ciencias de la Salud, INTEC. 
Se olvida que una gran parte de la población de los países en desarrollo en la realidad usa remedios caseros de origen vegetal todo el tiempo y al enfermarse y visitar un médico tomarán la medicina recetada por éste junto a, o previo, o después de utilizar las sustancias de origen vegetal a que están acostumbrados. Reyes y $\mathrm{Mármol}^{4}$ informan en su trabajo que de una muestra de más de mil personas tomadas en la ciudad de Santo Domingo, el $34 \%$ prefería los remedios caseros a los recetados, y de 45 enfermedades comunes, en el $73.34 \%$ de ellas las personas tratan de resolver el problema con fitoterapia, antes de ir al médico, en dos localidades dominicanas.

La necesidad del estudio científico de las plantas usadas por la población viene a tener entonces una doble justificación: Por un lado sus potenciales usos en la terapéutica científica y por el otro la necesidad de conocer si en realidad causan efectos que pudiesen interactuar con medicamentos recetados o si pudiesen entonces utilizarse en ciertas áreas como sustitutos prácticos de éstos.

En nuestro país se han realizado varios estudios sobre plantas medicinales. Algunos sobre identificación y valoración de componentes activos ${ }^{6,7}$ otros sobre entrevistas y encuestas a usuarios, vendedores y recolectores ${ }^{8,9}$ y otros sobre aspectos de divulgación de la medicina folklórica. ${ }^{10,11,12}$ En nuestra literatura aparecen pocos estudios experimentales sobre los efectos de las plantas y/o sus partes correspondientes en sistemas orgánicos específicos.

El presente trabajo informa acerca de los efectos inhibidores de infusiones de hojas de lectuca sativa o lechuga de ensalada, Annona muricata o guanábana y Tamarindus indica o tamarindo, sobre los parámetros de la conducta de ratones en "campo abierto". (Todos de mucho uso en ciertos estratos de nuestra población para las llamadas afecciones "nerviosas").

\section{Materiales y métodos}

Con el objeto de utilizar los compuestos en la forma más parecida posible a su uso popular se prepararon infusiones de hojas frescas de las plantas citadas a diferentes concentraciones. Se hirvió un litro de agua destilada y al iniciar la ebullición se agregó 10, 25, 50 o $100 \mathrm{gr}$. de hojas cortadas frescas y se dejaron hervir por 5 minutos a fuego lento. Luego se filtraba la infusión y se utilizó un $\mathrm{ml}$ inyectado intraperitonealmente en ratones blancos.

Se utilizaron varios grupos de animales, y siempre se utilizó como control un grupo que no había sido inyectado; otro grupo inyectado con un $\mathrm{ml} \mathrm{de}$ solución salina al $0.9 \%$ y otro grupo que se inyectaba y luego se retiraba la aguja sin introducir ninguna sustancia.

Los animales usados pesaban todos entre 20 - 25 gr. y fueron machos, obtenidos de los laboratorios del CESDA, de la Secretaría de Estado de Agricultura, en San Cristóbal. 
El estudio conductual se realizó en un "campo abierto" lo que permite estudiar una serie de parámetros como locomoción, conducta investigativa, miedo incondicionado y otros, paradigma muy estudiado en la literatura ${ }^{13,14,15}$ y de mucho uso en estudios con plantas medicinales. ${ }^{16,17}$

El "campo abierto" consistió en una caja de paredes de cartón-piedra y base metálica, de $465 \mathrm{~mm} \times 370 \mathrm{~mm} \times 270 \mathrm{~mm}$, con el interior pintado de amarillo y la base dividida en cuadros de $120 \times 120 \mathrm{~mm}$ cada uno. Sobre el centro se instaló una bombilla de $15 \mathrm{~W}$ a $300 \mathrm{~mm}$ de altura. Los animales eran colocados en uno de los cuadros del centro $15 \mathrm{~min}$. después de ser inyectados y visualmente dos observadores registraban el número de cuadros recorridos, el número de paradas sobre patas traseras, el número de veces que se acicalaban y el número de defecaciones durante un período de 5 minutos.

Los experimentos se repitieron cada 24 horas 5 ó 3 veces por grupo.

En total se utilizaron 200 ratones.

Los resultados se analizaron utilizando análisis de varianzas y pruebas " $T$ " de student.

\section{Resultados}

Es de interés mostrar los resultados de las comparaciones entre los grupos considerados controles, como son animales no manipulados, animales inyectados con solución salina y animales a los cuales se les introdujo la aguja de la jeringuilla o sea, que reciberon un pinchazo solamente.

En campo abierto los tres grupos fueron disminuyendo su actividad a medida que pasaban las pruebas. Tanto en las medidas de locomoción como de paradas sobre los cuartos traseros (investigación) los ratones que recibieron un pinchazo solamente fueron superiores a los otros dos grupos de manera significativa. También el grupo con inyección de solución salina recorrió más cuadros y se detuvo y elevó sobre sus patas traseras más veces que los controles sin ninguna manipulación.

En el número de defecaciones por prueba, una medida de neofobia, los tres grupos controles no presentaron diferencias significativas entre sí.

Al comparar los datos de los animales experimentales inyectados con extractos de lechuga fresca, grupo I ( $10 \mathrm{~g} / 1), \mathrm{II}(25 \mathrm{~g} / 1)$ y III $(50 \mathrm{~g} / 1)$ y los animales inyectados con solución salina se observa que en la primera prueba los animales del grupo III (un ml de solución de $50 \mathrm{~g} / 1$ ) fueron los menos activos de todos con una media recorrida de $42.46 \pm 8.67$ medida que es significativamente inferior $(P<0.05)$ a la de los grupos I y II y a la de los controles. Los grupos I y II sólo presentan una disminución significativa con respecto a los controles en las dos pruebas finales (luego de tres pruebas, interdiarias). 
En las paradas sobre cuartos traseros durante la observación en el campo abierto sólo los grupos II y III, inyectados con un ml de concentraciones de 25 y $50 \mathrm{~g} / \mathrm{l}$ mostraron diferencia significativa con los controles $(\mathrm{P}<0.05)$, con promedios totales y desviaciones estandard de $35.6 \pm 6.20$ para los controles y de 21.4 \pm 5.01 y 13.2 \pm 4.71 para los grupos I y II respectivamente. Se observa un aumento significativo de inhibición de acuerdo a la dosis.

La cantidad de movimientos de acicalamiento también mostró una disminución significativa en los grupos inyectados con extracto de lechuga en comparación con los controles, aunque esta diferencia no se da en el número de defecaciones.

En el estudio de los extractos de guanábana se observó una disminución significativa de la locomoción en el campo abierto de los grupos IV y V (50 y $75 \mathrm{~g} / 1)$ y en el grupo VI $(100 \mathrm{~g} / 1)$ ésta fue muy significativa $(P<0.01)$, con promedios totales de $38.5 \pm 9.31,27.0 \pm 9,56$ y $20.0 \pm 7.28$ respectivamente contra $114.9 \pm 12.5$ de los grupos controles.

También los extractos de las hojas de tamarindo $(15,25$ y $50 \mathrm{~g} / 1)$ disminuyeron significativamente los parámetros locomotores, tanto en comparación con los controles, como con los grupos de lechuga y guanábana, con valores totales entre $4.52 \pm 3.42$ para los grupos que recibieron $1 \mathrm{ml}$ de extracto de $50 \mathrm{~g} / 1$ y $10.4 \pm 8.2$ para el grupo de $15 \mathrm{~g} / 1$.

En los estudios del número de acicalamientos y de paradas se observa una repetición de este fenómeno de aumento de inhibición. Los tres extractos usados reducen significativamente estos parámetros en los test de campo abierto, en los animales estudiados, con una mayor inhibición lograda por los extractos de tamarindo.

Todos los animales estudiados disminuían sus indicadores en el campo abierto a medida que se repetían los pruebas, tanto los controles como los experimentales con la excepción del acicalamiento. En esta conducta tanto los controles como los animales inyectados con extractos de tamarindo y guanábana aumentaban sus movimientos de acicalamiento en pruebas sucesivas del campo abierto. Sólo el extracto de lechuga produjo una disminución en acicalamiento entre el primer día y el resto de las pruebas $(P<0.05)$. Ver gráfica1.

La gráfica 2 y el cuadro 1 nos muestran el efecto de los extractos estudiados, a ciertas concentraciones, sobre actividades de ratones en campo abierto.

Ninguno de los extractos afectó la deposición de bolos por los ratones en el e sperimento.

Por otro lado, al terminar las pruebas y a veces durante estas, los animales in rectados con tamarindo se dormían y aunque no se cuantifić el esfuerzo por despertarlos, al moverlos y despertarlos se quedaban en la misma posición y la mayoría de las veces sin intentar trasladarse de lugar. 
Durante toda la investigación no se notaron cambios notables en el peso de los animales, aunque sí parece existir una ligera tendencia hacia el aumento de peso en los animales tratados con guanábana y tamarindo.

\section{Discusión}

Los resultados presentados señalan que en las tres plantas estudiadas existen compuestos neuroactivos que inhiben la conducta exploratoria de ratones en el campo abierto. Debe recordarse que nuestros ratones son ya animales excitados, pues los resultados también señalan diferencias entre los controles inyectados y pinchados con los no manipulados de manera estresante.

Por los resultados también notamos que si bien los extractos estudiados inhiben ciertos parámetros específicos, como son locomoción, conducta investigativa y acicalamiento; no se alteran ni la defecación en campo abierto, que se tiene como medida de neofobia ${ }^{18}$ ni la memoria, pues se considera la disminución progresiva de las medidas en campo abierto como un indicador de recuerdo de la situación experimental. ${ }^{13}$

El extracto de hojas de tamarindo fue el más activo de todos y aunque en la literatura se reporta el uso de la pulpa de este fruto como laxante, en toda la cuenca del Caribe, ${ }^{19}$ no influyó en el número de defecaciones en el experimento. Debemos recordar que nuestra manipulación experimental ocurre 15 minutos luego de la inyección y la observación sólo durante 5 minutos; por lo que el efecto laxante puede no manifestarse en este tiempo.

La literatura señala el uso del te de lechuga ${ }^{20,21,22}$ como somnífero y calmante, indicando el lactucario, líquido lechoso que se extrae del tallo como el agente causante de este efecto. Aunque aparece raramente en las encuestas publicadas en nuestro país sobre medicina tradicional, las obras de divulgación la presentan como de amplio uso. El autor ha recibido comunicaciones personales sobre su uso para inducir el sueño a niños.

La guanábana aparenta ser la más estudiada de las tres especies. En ella se han identificado alcaloides, esteroides, terpenoides, flavonoides, compuestos fenólicos y taninos, y los efectos de esos extractos se han estudiado sobre varios sistemas y se recomienda su estudio sobre la conducta y el SNC. ${ }^{19}$

La rapidez de los efectos de las infusiones señalan que él o los productos causantes de tales efectos no tienen que ser metabolizados para actuar sobre el SNC.

Tanto los ansiolíticos como los neurolépticos disminuyen la locomoción en experimentos en campo abierto, especialmente en dosis de moderadas a altas, ${ }^{16}$ por lo que los compuestos estudiados presentan una acción parecida.

Es interesante discutir el fenómeno del acicalamiento. 
Si bien los tres extractos estudiados disminuyen esta actividad, sólo la solución de las hojas de lechuga la disminuye también en cada prueba.

File et al. ${ }^{23}$ obtuvo resultados similares a los nuestros, donde en cada sesión de campo abierto, el acicalamiento aumentaba, y propone que dicha conducta puede ser una respuesta a la habituación a estímulos ansiogénicos. Además expresa, que dadas condiciones iguales de habituación el estímulo más ansiogénico generaría más acicalamiento, por lo que la disminución del aumento progresivo del acicalamiento por el extracto de lechuga puede servir para catalogar a ésta como ansiolítico y quizás deducir que los extractos de tamarindo y guanábana sean neurolépticos.

Varios autores sugieren que el fenómeno de acicalamiento depende de vías nerviosas dopaminérgicas y de receptores opiáceos, así como también del GABA y el 5-HT que interactúan con dichas vías. ${ }^{24,25,26,27}$

Utilizando ratas, Kametani ${ }^{28}$ demostró que animales jóvenes presentan un aumento de acicalamiento en campo abierto a medida que aumentan las pruebas, pero que este aumento era significativamente menor que el presentado por ratas de más edad, lo que significa en nuestra prueba que en animales jóvenes el extracto de lechuga tendría un mayor efecto y quizás esto muestre la utilización del extracto en niños por parte de la medicina tradicional.

Nuestro estudio sólo observó períodos de acicalamiento durante cinco minutos en campo abierto, 15 minutos después de las inyecciones de los extractos. No se analizaron los patrones finos de esta conducta ni su secuencia motora. Este estudio nos daría datos más precisos sobre la influencia de estos compuestos en el acicalamiento.

El efecto interesante de la tisana de lechuga amerita ser estudiado más profundamente, ya que señala un mecanismo de acción distinto a las otras dos infusiones estudiadas.

En resumen, nuestro trabajo presenta un primer acercamiento a la comprensión de los efectos de extractos de hojas de tamarindo, guanábana y lechuga sobre la conducta de ratones de laboratorio. Los resultados muestran efectos tranquilizantes e inhibidores sobre varios parámetros conductuales. Se precisa entonces profundizar estos conocimientos así como investigar los efectos de interacción de estos extractos, tanto con sustancias farmacológicas experimentales, como con aquellas de uso clínico común, que actúan sobre el SNC. 


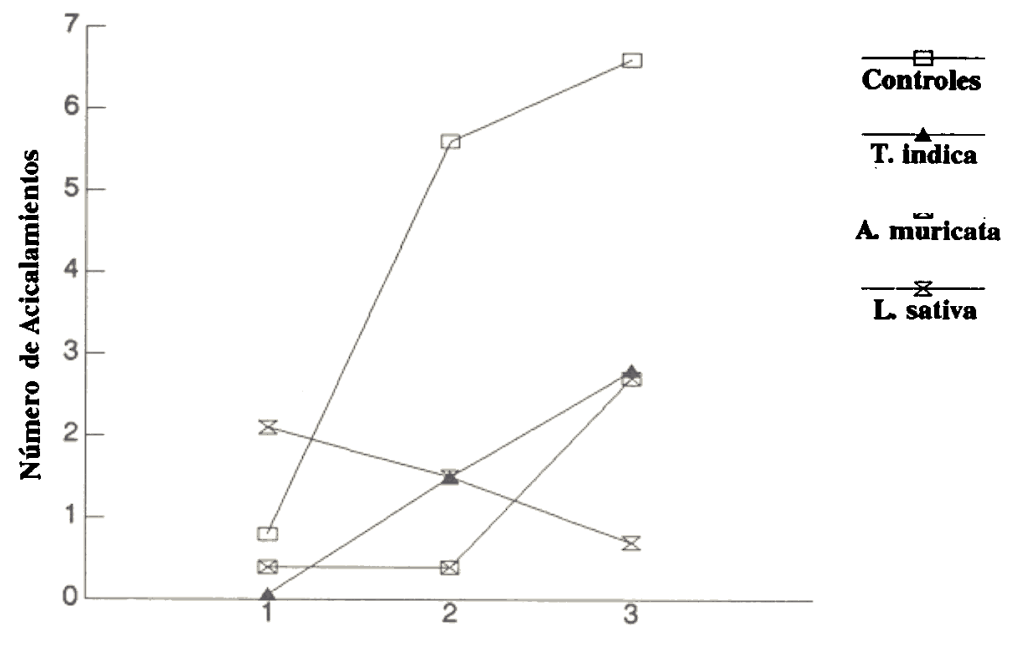

Dia de Ensayos

GRAFICOI. Número de patrones de acicalamiento en ratones inyectados con $1 \mathrm{ml}$ (ip) de extractos de hojas frescas de las plantas estudiadas, en campo abierto durante $5 \mathrm{~min}$. en tres días consecutivos. 


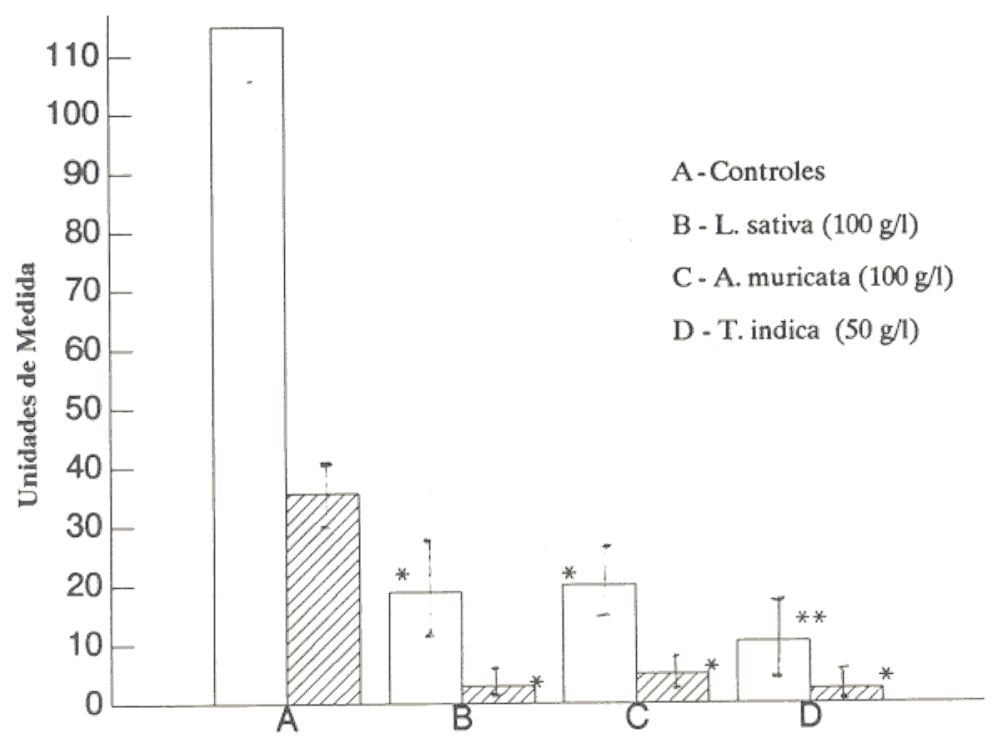

GRAFICOII.Cuadros recorridos $\square$ y paradas y elevación UIIA de ratones en campo abierto bajo los efectos de $1 \mathrm{ml}$ (ip) de infusiones delechuga, L. sativa,ganábana,A. muricataytamarindo, T. indica adistintasconcentraciones.

Totales de tres días de pruebas.

${ }^{*} \mathrm{P}<0.05$

$* * \mathbf{P}<0.001$ 


\section{CUADRO 1}

RESULTADOS TOTALES DE LA CONDUCTA DE RATONES EN CAMPO ABIERTO, INYECTADOS CON 1ML (IP) DE EXTRACTOS DE HOJAS FRESCAS DE LECHUGA (L. SATIVA), GUANABANA (A. MURICATA) Y TAMARINDO (T. INDICA) A LA CONCENTRACION DE 50G/1

\begin{tabular}{lccccc} 
& $\mathrm{N}$ & $\mathrm{C}$. recorridos & Paradas & Acicalamiento & Defecaciones \\
\hline CONTROL & 21 & $114 \pm 12.5$ & $35.6 \pm 6.20$ & $4.4 \pm 2.70$ & $0.75 \pm 0.39$ \\
L. Sativa & 14 & $42.46 \pm 8.6^{*}$ & $13.20 \pm 4.71^{*}$ & $2.0 \pm 1.0^{*}$ & $1.0 \pm 0.70$ \\
A. muricata & 14 & $38.5 \pm 9.31^{*}$ & $14.6 \pm 8.70^{*}$ & $1.2 \pm 1.60^{*}$ & $0.4 \pm 0.42$ \\
T. indica & 14 & $10.4 \pm 8.42^{* *}$ & $2.4 \pm 3.48^{* *}$ & $1.1 \pm 0.76^{* *}$ & $0.9 \pm 0.8$ \\
\hline
\end{tabular}

*P $<0.05 ;{ }^{* *} \mathrm{P}<0.001(\overline{\mathrm{X}} \pm$ D.E. $)$ 


\section{LITERATURA CITADA}

1. W.H.O. (1983). Tradicional medicine and health care coverage. Macmillan, Geneva. 342 págs.

2. De Smet, Peter A. G. M. y L. Rivier (1989). A general outlook on etnopharmacology. Jour. of Etnopharm. 25: 127-138.

3. Phillipson, J. David y L. A. anderson (1989). Etnopharmacology and western medicine. Jour. of Etnopharm. 25: 61-72.

4. Reyes, M., D. Montero y E. Mármol. (1981). Medicina tradicional y científica. Ciencias y prácticas en la ciudad de Santo Domingo. (tesis). Escuela de Medicina. Fac. Ciencias de la Salud. UASD. 243 págs.

5. Robineau, L. (1986). Encuesta sobre medicina tradicional popular en una zona rural y una zona urbana marginal de la República Dominicana. MOSCOSOA, 4: 226-265.

6. Acosta E. (1968). Extracción, identificación y valoración de componentes activos de plantas medicinales determinando el porcentaje requerido oficial (Encajes de la F.E.U.F.N.E.U.). (Tesis). Depto. de Farmacia. Facultad de Ciencias, UASD. 41 Págs.

7. Vásquez Tineo, M. (1986). Métodos químico-biológicos para investigar plantas medicinales. V Jornada de Biología sobre Botánica y Etnobotánica. Academica de Ciencias de la República Dominicana.

8. Almonte, C. y J. Peña (1973). Las plantas en la medicina popular: Propiedades y usos. (Tesis). Depto. de Farmacia, Facultad de Ciencias, UASD. Santo Domingo. 43 págs.

9. Hernández Colón, S. (1976). The traditional use of medical plants and herbs in the province of Pedernales, Santo Domingo, República Dominicana. Ethnomed, IV (1,2): 139-165.

10. Cordero, A. (1978). Manual de Medicina Doméstica. Ed. UASD. Santo Domingo. 490 págs.

11. Sánchez, M., F. (1980). Medicina Folklórica. Colec. SESPAS; Santo Domingo, 59 págs.

12. Mañón, R. W. (1975). Apuntes de medicina folklórica en República Dominicana. (Tesis). Depto. de Medicina, Facultad de Salud, UASD. 91 págs.

13. Albaine Pons, J. R. y G. Chaichenko. (1980). Effects of basolateral and corticomedial part of amygdala of rats on openfield behaviour. Phisiologichesky Jour. Ukranian Acad. of Scienc. 26 (2): 261-264.

14. Katz, R. J.; K. A. Roth and B. J. Carrol. (1981). Acute and chronic stress effects on open-field activity in the rat: Implication for a model of depression. Neurosci Biobehav. Rev. Vol. 5: 247-251. 
15. Albaine Pons, J. R. (1982). Participación del hipotálamo ventromedial en la integración del comportamiento alimenticio y defensivo en ratas. Ciencia (UASD). IV (1-4): 11-28.

16. Fernández, L., H. Pérez Saad; R. Mas et al. (1987). Efecto de Justicia pectoralis sobre la conducta exploratoria de ratones. En: Estudios avanzados en neurociencias. La Habana, Cuba: 257-264.

17. Sattar, M.A.; E. K. Gan; S. E. Loke et al. (1989). Effect of an extract of Erioglossum edule on the central nervous system. Jour of Etnopharmacol. 25: 217-220.

18. Walsh, R. N. and R. A. Cummins. (1976). The open-field test. A critical review. Psicol. Bull. 83: 482-504.

19. Seminario TRAMIL - 2 (1986). (D. Werniger y L. Robineau, ed.) Enda Caribe - Santo Domingo. 255 págs.

20. Sarita, V. V. (1989). Olericultura. Ed. Taller, Santo Domingo. 464 págs.

21. Font Quer, P. (1978). Plantas medicinales. Ed. Labor. Barcelona. 1033 págs.

22. Zin, J. y C. Weiss. (1980). La salud por medio de las plantas medicinales. 6ta. ed. Ed. Salesiana. Santiago, Chile. 387 págs.

23. File, S. E.; P. S. Mabbutt and J. H. Walker. (1988). Comparison of adaptative responses in familiar and novel environments: Modulatory factors. En: Neural Mechanisms and Biological Significance of Grooming Behaviour (D. C. Colbern, W.H. Gispen, eds.). Ann. N.Y. Acad. Sci. Vol. 525: 69-79.

24. Dunn, A, J. (1988). Studies on the neurochemical mechanisms and significance of ACTH-induced grooming. En: Neural Mechanisms and Biological Significance of Grooming Behaviour (Colbert \& Gispen, eds.) Ann. N.Y. Acad. Sci., Vol. 525: 150-168.

25. Kulkosky, P. J. (1988). Bombesin and ceruletide-induced grooming and inhibition of ingestion in the rat. En: Neural Mechanisms and Biological Significance of Grooming Behavior. (Colbert \& Gispen, eds.) Ann. N. Y. Acad. Sci., Vol. 525: 321-329.

26. Isaacson, R. L. (1988). Excessive grooming in response to opiate drugs. En: Neural Mechanisms and Biological Significance of Grooming Behavior. (Colbern \& Gispen, eds.) Ann. N.Y. Acad. Sci., Vol. 525: 338-349.

27. Cools, A. R.; B. M. Spruigist and B. A. Ellenbrok. (1988). Role of central dopamine in ACTH-induced grooming in rats. En: Neural Mechanisms and Biological Significance of Grooming Behavior. (Colbert \& Gispen, eds.), Ann. N. Y. Acad. Sci., vol. 525: 338-349.

28. Kametami, Hideki. Analysis of Age-Related Changes in Stress-induced Grooming in the Rat: Differential behavioral profile of adaptation to stress. En: Neural Mech. and Biol. Significance of Grooming Behav. (Colbert \& Gispen, eds.) Ann. N. Y. Acad. Sci., vol. 525: 101-113. 